\title{
Inhalation of Bacterial Cellulose Nanofibers Facilitates Silica Particle Clearance in a Rat Silicosis Model
}

\author{
Wen-Chuan $\mathrm{Li}^{1}$, Nai-Fang Fu ${ }^{2}$, Jun-Cai Wu ${ }^{2}$, Xian-Jun Li ${ }^{3}$, Rui-Hui Pan ${ }^{3}$, Yan-Yan Zheng ${ }^{3}$, \\ Yong-Jin Gan ${ }^{3}$, Jian-An Ling ${ }^{3}$, Heng-Qiu Liang ${ }^{3}$, Dan-Yu Liang ${ }^{3}$, Jing Xie ${ }^{3}$, Zhi-Chao Dong ${ }^{3}$, \\ Sheng-Jun Jiang ${ }^{2,4}$, * \\ ${ }^{1}$ Graduate College of Youjiang Medical College for Nationalities, Baise, China \\ ${ }^{2}$ Tropical Crop Genetic Resources Institute, Chinese Academy of Tropical Agriculture Science, Danzhou, China \\ ${ }^{3}$ Guangxi Zhuang Autonomous Region Institute for Chemical Toxicity Testing, Guangxi Zhuang Autonomous Region Academy for the \\ Prevention and Treatment of Occupational Disease, Nanning, China \\ ${ }^{4}$ Hainan Mulada Bio-science \& Bio-technology Co., Ltd, Haikou, China
}

\section{Email address: \\ 18708938111@139.com (Sheng-Jun Jiang) \\ *Corresponding author}

\section{To cite this article:}

Wen-Chuan Li, Nai-Fang Fu, Jun-Cai Wu, Xian-Jun Li, Rui-Hui Pan, Yan-Yan Zheng, Yong-Jin Gan, Jian-An Ling, Heng-Qiu Liang, Dan-Yu Liang, Jing Xie, Zhi-Chao Dong, Sheng-Jun Jiang. Inhalation of Bacterial Cellulose Nanofibers Facilitates Silica Particle Clearance in a Rat Silicosis Model. American Journal of Clinical and Experimental Medicine. Vol. 4, No. 6, 2016, pp. $204-211$. doi: 10.11648/j.ajcem.20160406.18

Received: November 7, 2016; Accepted: November 22, 2016; Published: November 24, 2016

\begin{abstract}
Background: Millions of workers globally are afflicted by pneumoconiosis, a disease caused by inhaling dust or particles. A particularly prevalent form is silicosis, caused by inhaling silica particles. The Chinese herbal medicine kombucha, which contains Gluconacetobacter xylinus and yeasts, can effectively clear dust from rats' lungs. The study presented here assessed the potential effectiveness of inhalable bacterial cellulose nanofibers (IBCNs) prepared from G. xylinus cultures for facilitating clearance of silica particles in a rat silicosis model. Methods: For this purpose, $50 \mathrm{mg}$ portions of silica dust were injected into lungs of rats, which were subsequently exposed to IBCNs for a month. The treatment's effects were then evaluated by examining the extent and severity of histopathological lesions in the animals' lungs, analyzing gas contents of blood samples, and determining organ coefficients, lung collagen contents, lungs' dry and wet weights, silica particle clearance rates, and both numbers and types of cells in lung lavage fluid. Results: IBCN inhalation was found to relieve the detrimental effects of silica exposure and facilitated silica particle clearance in a rat silicosis model. Unexpectedly, our results also indicated that saline inhalation also strongly stimulates silica particle clearance from rat lungs. Conclusions: These results provide the first evidence for a functional effect of IBCN inhalation in a rat silicosis model, indicating that bacterial cellulose nanofiber inhalation can facilitate silica particle clearance. Further studies are required to determine whether these effects are mediated by IBCN and define the mechanisms involved. The findings also indicate that salt water may effectively clear dust from lungs, thereby alleviating risks of silicosis and reducing risks associated with haze and smog.
\end{abstract}

Keywords: Inhalable Bacterial Cellulose Nanofibers, Rat, Silica Particle, Silicosis, Saline Water

\section{Introduction}

Pneumoconiosis afflicts millions of workers globally. Silicosis, caused by inhaling silica particles, is a particularly prevalent form, but it can be caused by inhaling diverse kinds of dust or particles [1]. The Chinese herbal medicine kombucha is a promising source of pneumoconiosis therapeutics because it can effectively induce removal of dust from rats' lungs [1] and has demonstrated efficacy for treating gold pneumoconiosis [2]. It is prepared by fermenting Chinese herbal extracts with a kombucha culture, which contains Gluconacetobacter xylinus and yeasts [3]. Bacterial cellulose secreted by the G. xylinus in kombucha cultures has several useful properties for medical applications, including high biocompatibility, thinness (the 
sheets are typically only $0.1 \mu \mathrm{m}$ thick), high specific surface area and very high capacity for adsorbing toxic heavy metal ions (e.g. $\mathrm{Cu}^{2+}, \mathrm{Pb}^{2+}, \mathrm{Hg}^{2+}$, and $\left.\mathrm{Cd}^{2+}\right)$ and non-metallic toxins such as histamines, ammonia, $\mathrm{NO}^{2-}$ and formaldehyde [4-10].

We hypothesized that at least some of the apparent beneficial effects of kombucha for treating silicosis and other forms of pneumoconiosis are due to its bacterial cellulose content. To test this hypothesis and hopefully pave the way to develop new curative or preventative agents against silicosis, we investigated effects of spraying IBCN prepared from G. xylinus cultures into lungs of rat silicosis models. We anticipated that the bacterial cellulose would adsorb the mineral dust in the animals' lungs and facilitate its clearance.

\section{Materials and Methods}

\subsection{Preparation and Analysis of IBCN}

To avoid contaminating the $\mathrm{BC}$ with other materials, $\mathrm{BC}$ membranes were prepared from cultures of $G$. xylinus, which was isolated from kombucha then statically cultivated in $20 \mathrm{ml}$ portions of nutrient medium in $100 \mathrm{ml}$ flasks for $120 \mathrm{~h}$ at $28^{\circ} \mathrm{C}$. The nutrient medium contained (w/w) 2\% glucose, $0.5 \%$ peptone, $0.5 \%$ yeast extract, $0.27 \%$ disodium hydrogen phosphate and $0.115 \%$ citric acid. BC pellicles were harvested and cleaned by immersion in $2 \%(\mathrm{w} / \mathrm{w}) \mathrm{NaOH}$ solution at $80^{\circ} \mathrm{C}$ for $1 \mathrm{~h}$. The pellicles were then immersed in $1 \%(\mathrm{w} / \mathrm{w}) \mathrm{NaClO}$ solution for $30 \mathrm{~min}$, washed with deionized water and sterilized by autoclaving [11]. Because only inhalable materials can be taken up into lungs, inhalable BC nanofibers were prepared by disrupting the sterilized pellicles using a Supor DJ13B-21G beating machine (Zhejiang Supor Co., Ltd., China), followed by homogenization using a AH-100D high pressure homogenizer (ATS Engineering INC Co., Ltd., Canada) for 1, 10, or 30 cycles at $80 \mathrm{MPa}$. The inhalable nanofiber $(\mathrm{PM}<10 \mu \mathrm{m})$ contents of the resulting homogenized materials were around $1 \%, 2 \%$, and $3 \%$ $(\mathrm{v} / \mathrm{v})$, respectively. The particle sizes and size distributions of the homogenized materials were determined using a Mastersizer 2000 (Malvern Instruments, Malvern, UK). The surfaces of the homogenized BC materials were examined using a JSM-7500F field-emission-type high-resolution scanning electron microscope (SEM) from JEOL (Japan).

\subsection{Experimental Animals}

Pathogen-free Sprague-Dawley rats were purchased from Hunan Slack Jingda (China). All rats were held under quarantine for 7 days to adjust to their new environment before the experiments began. The 120 rats used in the experiments reported here each initially weighed 180-220 g and groups of 24 (12 male and 12 female) were randomly assigned to five treatments designated $1 \%$ IBCN inhalation, $2 \%$ IBCN inhalation, 3\% IBCN inhalation, $\mathrm{P}$ (positive control), and $\mathrm{N}$ (negative control). Silica particles were injected into the trachea of rats of the IBCN inhalation and $\mathrm{P}$ groups, then the IBCN inhalation groups were exposed to homogenized $\mathrm{BC}$ with the indicated nanofiber content, while the $\mathrm{P}$ group was exposed to a saline solution and the $\mathrm{N}$ group was exposed to water in the same manner. All groups were fed standard rodent laboratory chow and allowed to eat add libitum during the study. The rats were housed under 12-hour photoperiods in a temperature-controlled room at $26^{\circ} \mathrm{C}$. All experiments were approved by the Ethics Review Committee of the Academy of Occupational Disease Prevention, Guangxi, China.

\subsection{Tracheal Injection of Silica Particles and Exposure of Animals to IBCN}

Silica particles $(99 \%$ with diameters of $0.5-10 \mu \mathrm{m}$ and $80 \%$ with diameters of $1-5 \mu \mathrm{m}$ ) were purchased from Sigma Aldrich (USA). A $50 \mathrm{mg} / \mathrm{ml}$ standard suspension of silica particles in saline was prepared. Prior to tracheal injection, samples of the stock solution were autoclaved then mixed with mycillin $(4000 \mathrm{U} / \mathrm{mL})$. The experimental animals were then anaesthetized with ethyl ether under sterile conditions and all except the negative controls were subjected to intra-tracheal injection with $1 \mathrm{ml}$ of the sterile mycillin-containing silica particle suspension [12]. Each rat in the $1 \%, 2 \%$, and $3 \%$ IBCN inhalation treatment groups and positive group was thus injected with $50 \mathrm{mg}$ of silica particles, while each rat in the negative group was injected with $1 \mathrm{ml}$ of salt water. Then twice daily for 30 consecutive days, starting three days after the injection of silica particles, each rat in the IBCN inhalation groups was sprayed with an aerosol containing homogenized BC with the appropriate IBCN content for one minute. Rats in the positive and negative control groups were sprayed with saline solution during this period in the same fashion: using fine sprays (of ICBN or saline solution) generated with a Yuyue 402AI ultrasonic nebulizer and nozzle (Jiangsu Yuyue Medical Equipment Ltd., Jiangsu, China) [13-15].

\subsection{Determination of Organ Coefficients and Weights (Wet and Dry) of Lung Tissues}

At the end of the treatment period, all of the animals subjected to each treatment were killed by arterial bloodletting from the groin. Their hearts, livers, spleens, kidneys, and other organs and tissues were then immediately removed and weighed, and the organ coefficient for each organ was calculated as a percentage of body weight. The trachea and lungs were then removed and separated, and the lungs were stripped of their connective tissue. The connective tissues were soaked in water and the wet lung weight (M) was measured. A $0.5 \mathrm{~g}$ lung tissue sample was then degreased by immersion in acetone for three days, cut into pieces, baked in an oven at $105^{\circ} \mathrm{C}$ for $12 \mathrm{~h}$, and weighed to determine its dry weight $(\mathrm{m})$. The dry weight of the lung was then estimated as $2 \mathrm{~m} \times \mathrm{M}$. Silica particles induce production of collagen around them, causing lung tissue proliferation. Therefore, the proliferation of lung tissues is proportional to the quantity of silica particles inhaled and the initial lung weight. 


\subsection{Counting and Classification of Cells in Lung Lavage Flid}

Sets of 12 rats subjected to each treatment (6 male and 6 female) were killed by arterial bloodletting from the groin. The trachea was then removed and a V-shaped opening was made in the lower trachea (roughly a third of the way between the bifurcation of the trachea and larynx) of each of these rats. One end of a small plastic hose was fitted with an eight-gauge needle and the other end was inserted into the V-shaped opening and ligated to the lung using fixed lines. $5 \mathrm{ml}$ of saline was taken up in a syringe, which was then attached to the needle at the end of the tubing. The saline was slowly injected into the alveoli and then slowly withdrawn, yielding approximately $3 \mathrm{ml}$ of recovered liquid [16]. The cells in the resulting bronchoalveolar lavage fluid were collected by centrifugation at $1,000 \times g$ for 10 min and re-suspended in phosphate-buffered saline ( $\mathrm{pH}$ 7.4). After counting the total number of cells, they were observed in a Giemsa-stained smear on a glass slide under a BX51 light microscope (Olympus Co., Tokyo, Japan).

\subsection{Pathological Analysis of Lung Tissues}

To examine pathological effects of the treatments on the rats' lungs, the gross morphology of lungs obtained from another set of 6 rats killed by arterial bloodletting was first examined by direct visual inspection. The lung tissue remaining after taking the sample used for dry weight determination was then fixed using formalin, conventional paraffin sections were taken and stained with hematoxylin-eosin. The stained tissue sections were inspected for pathological changes and the presence of nodules using a Model BX43 optical microscope (Olympus Co., Tokyo, Japan). In addition, the tissue sections' collagen fiber contents were determined, along with their distribution and extent of reticular fiber hyperplasia. The results obtained were evaluated according to the diagnostic criteria for pneumoconiosis specified in the Chinese national occupational health standards $[1,15]$.

\subsection{Determination of Lung Silicon Dioxide Clearance Rates}

The silica contents of whole lung samples obtained from another set of 6 rats were initially determined using the method presented in the Chinese national standard GBZ/T192.4-2007 ("Determination of dust in workplace air, Part 4: Content of silicon dioxide in dust") [1]. Briefly, fresh rat lung samples were degreased, dried, and crushed. Samples of the crushed material $(0.1000 \mathrm{~g})$ were then analyzed using the above method. The silica content of the lung samples (in units of $0.10 \mathrm{~g}$ ) was denoted using the variable $d$. The total silica contents of the lungs were then calculated as $10 \mathrm{~d} \times$ DFW (where DFW is total dry weight of the lung). Silica contents of the whole lung samples were subsequently calculated, the total quantity of silica removed from each sample was calculated by subtracting the resulting amount from the amount added in the treatment $(50 \mathrm{mg}$, for all groups except negative controls) + the background amount detected in negative controls $(\mathrm{mg})$. The rate of silica clearance from the lung during the 30-day ICBN- or saline-spraying period was then calculated in $\mathrm{mg} / \mathrm{d}$.

\subsection{Statistical Analysis}

Measured variables were recorded as means \pm standard deviations. The significance of between-treatment differences in the means was assessed by student's and paired t-tests, after evaluating the normality of the data distributions with the Shapiro-chi-square test. Differences with $P<0.05$ were considered significant. The SPSS version 17.0 software package (SPSS Inc., Chicago, USA) was used for all statistical analyses.

\section{Results}

\subsection{Characterization of the Prepared IBCN}
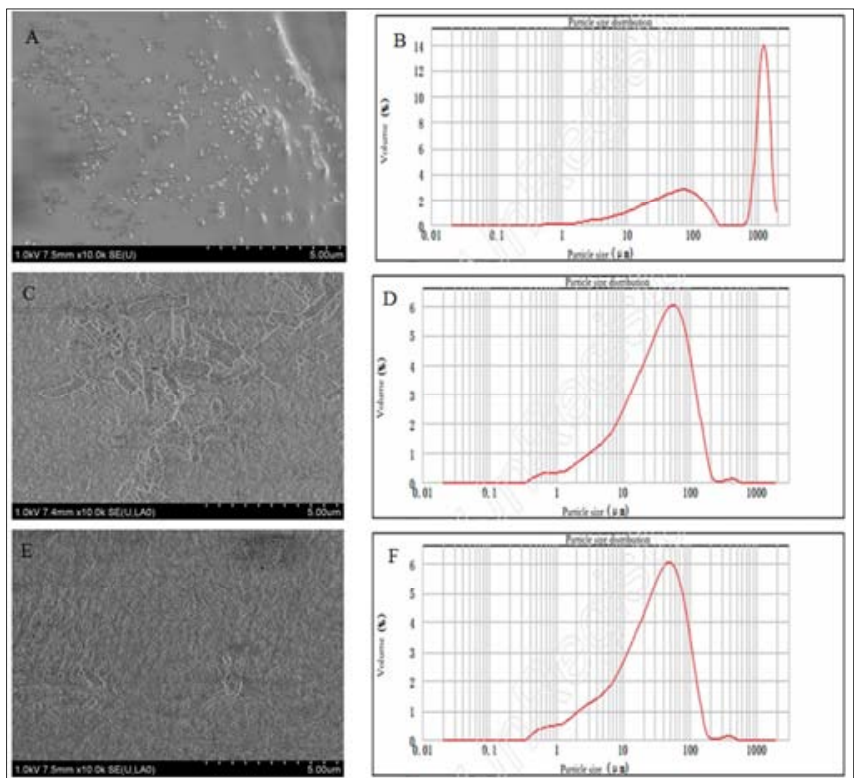

Figure 1. Physicochemical properties of BC nanofibers. SEM images and volume mean diameters of $B C$ nanofibers after one (A, B), $10(C, D)$ and 30 (E, $F)$ homogenization cycles. 
Bacterial cellulose (BC) membranes were broken into pieces in a beating machine and then homogenized in a high pressure homogenizer. The IBCN content of the homogenized material (i.e. the content of cellulose particles with $<10 \mu \mathrm{m}$ diameters) depended on the number of homogenization cycles performed: one, ten, and thirty cycles yielded IBCN contents of around $1 \%, 2 \%$, and $3 \%$ by volume, respectively (Figure 1). The IBCN content of the homogenized material (i.e. content of cellulose particles with $<10 \mu \mathrm{m}$ diameters) depended on the number of homogenization cycles applied: one, 10, and 30 cycles yielded IBCN contents of around $1 \%, 2 \%$, and $3 \%(\mathrm{v} / \mathrm{v})$, respectively (Figure 1). Thus, as expected more extensive homogenization reduced the size of the $\mathrm{BC}$ nanofibers and

increased the IBCN content.

\subsection{Rat Growth and General Health}

In the period immediately after tracheal injection with silica particles the rats exhibited symptoms of dyspnea, did not eat much, and were sluggish. However, they subsequently recovered and their body weights increased. All of the rats appeared healthy and behaved normally during the experimental period when they were treated with IBCN or saline via inhalation. There were no unplanned deaths among the rats in the silica-exposed and IBCN inhalation groups.

\subsection{Lung Pathology}
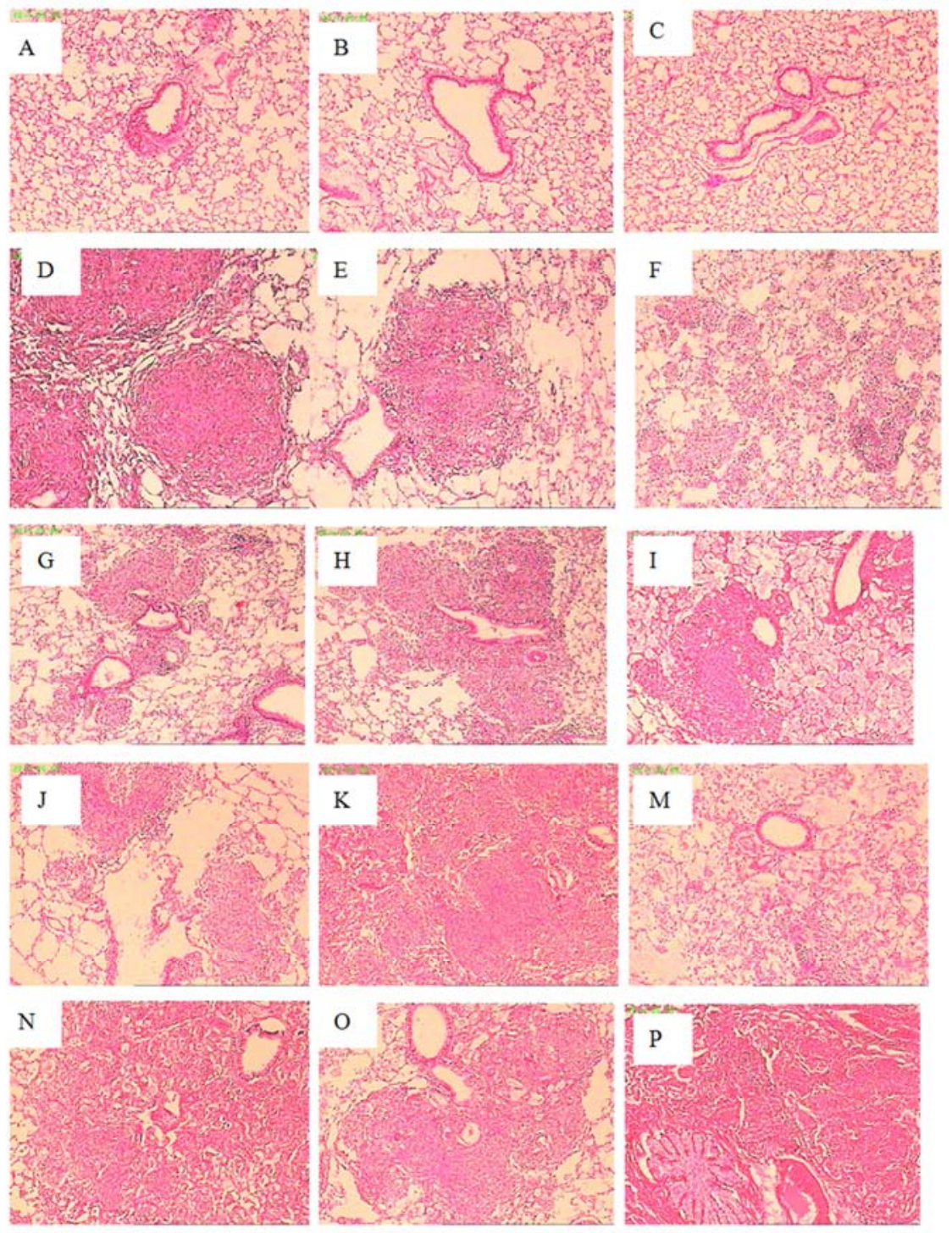

Figure 2. Hematoxylin- and eosin (H\&E)-stained sections of lungs of rats of: the negative control group (A-C), $1 \%$ IBCN exposure group (D-F), 2\% IBCN exposure group (G-I), 3\% IBCN exposure group $(J-M)$, and positive control group $(N-P)$. Original magnification $\times 40$.

Macroscopic inspection of the lungs of negative control group animals revealed that the two lobes and bronchi of the lungs were complete with no visible abnormalities. Microscopic inspection revealed that these pulmonary structures were clear with no pathological changes in the lobe bronchus, pulmonary segmental bronchus, bronchus, bronchioles, terminal bronchioles, alveolar ducts, or alveoli (Figure 2A-C). Macroscopic inspection of the lungs of rats 
from the $1 \%$ IBCN treatment group revealed complete lobes and bronchi with extensive or speckled surface tissue consolidation in some cases. Microscopic inspection showed that these pulmonary structures were clear; the tissue sections exhibited scattered or focal fibroblast nodules (Figure 2D, 2E), mild hyperemia, mild alveolitis and pulmonary edema pathology (Figure 2F). On average, tissue consolidation was visible over $22.5 \%$ of the lungs' surface area. Macro- and microscopic inspection of lung tissues from the positive control group and the $2 \%$ and $3 \%$ IBCN treatment groups yielded similar results to those for the $1 \%$ IBCN group (Figure 2G-P). The average percentages of lung tissue consolidation for the $2 \%, 3 \%$, and positive control groups were $20.0 \%, 22.5 \%$ and $20.0 \%$, respectively. In summary, the lung sections taken from animals in the $1 \%$ and $3 \%$ IBCN groups exhibited identical and relatively high levels of tissue consolidation, with pathologically severe lesion. In contrast, sections from the $2 \%$ ICBN and positive control groups exhibited somewhat less extensive tissue consolidation with more evidence of lung inflammation in sections from the $2 \%$ ICBN group.

\subsection{Organ Coefficients After Inhalation of IBCN or Saline}

At the end of the treatment period, the hearts, livers, spleens, lungs, and kidneys of rats in each group were removed and weighed, and the corresponding organ coefficients were calculated (Table 1). The average heart coefficient for the positive control group was significantly higher $(\mathrm{P}<0.05)$ than the coefficient for the negative control

Table 1. Effects of the IBCN inhalation and control treatments on the

\begin{tabular}{|c|c|c|c|c|c|}
\hline Organ $\backslash$ Group & $1 \%$ IBCN & $2 \%$ IBCN & $3 \%$ IBCN & Positive control & Negative control \\
\hline Heart & $0.34 \pm 0.03$ & $0.34 \pm 0.04$ & $0.33 \pm 0.05$ & $0.35 \pm 0.10^{*}$ & $0.31 \pm 0.04$ \\
\hline Liver & $2.96 \pm 0.34^{\Delta}$ & $2.88 \pm 0.32^{\Delta}$ & $2.97 \pm 0.40^{\Delta}$ & $3.39 \pm 0.79$ & $3.19 \pm 0.33$ \\
\hline Spleen & $0.26 \pm 0.08^{*}$ & $0.24 \pm 0.05$ & $0.25 \pm 0.04$ & $0.25 \pm 0.06$ & $0.21 \pm 0.03$ \\
\hline Lung & $1.26 \pm 0.30^{*}$ & $1.33 \pm 0.33^{*}$ & $1.23 \pm 0.24^{*}$ & $1.29 \pm 0.50^{*}$ & $0.46 \pm 0.11$ \\
\hline Kidney & $0.69 \pm 0.04$ & $0.70 \pm 0.04$ & $0.70 \pm 0.09$ & $0.73 \pm 0.06^{*}$ & $0.66 \pm 0.03$ \\
\hline
\end{tabular}

* and $\Delta$ : Significantly different from negative control group and positive control groups, respectively $(\mathrm{P}<0.05 ; \mathrm{n}=12)$.

\subsection{Cell Counts in Lung Lavage Fluid from Silica-Exposed Rats}

As shown in Table 2, the negative control and 1\% ICBN groups had the lowest and highest average lung lavage fluid cell counts $\left(0.10 \pm 0.03\right.$ and $1.18 \pm 1.12 \times 10^{9}$ cells $/ \mathrm{ml}$, respectively), while the $2 \% \mathrm{IBCN}, 3 \% \mathrm{IBCN}$, and positive control groups had intermediate counts $(0.58 \pm 0.32,0.57 \pm$

Table 2. Effects of the IBCN inhalation and control treatments on cell counts in the lung lavage fluid of rats exposed to silica dust particles (Mean \pm SD).

\begin{tabular}{|c|c|c|c|c|}
\hline \multirow{2}{*}{ Treatment } & \multirow{2}{*}{ Total cells $\left(\times 10^{9} / \mathrm{ml}\right)$} & \multicolumn{3}{|l|}{ Cell type (\%) } \\
\hline & & $\mathbf{N}$ & $\mathbf{L}$ & M \\
\hline $1 \% \mathrm{IBCN}$ exposure & $1.18 \pm 1.12^{*}$ & $7.42 \pm 3.97 *$ & $78.17 \pm 9.77^{*}$ & $14.42 \pm 8.11 *$ \\
\hline $2 \%$ IBCN exposure & $0.58 \pm 0.32$ & $18.25 \pm 12.06^{*}$ & $58.00 \pm 17.16^{*}$ & $23.75 \pm 9.55$ \\
\hline $3 \%$ IBCN exposure & $0.57 \pm 0.41$ & $10.00 \pm 7.52 *$ & $57.58 \pm 21.60 *$ & $32.42 \pm 24.49$ \\
\hline Positive control & $0.83 \pm 0.43^{*}$ & $11.92 \pm 6.11^{*}$ & $75.08 \pm 6.70^{*}$ & $13.00 \pm 8.81^{*}$ \\
\hline Negative control & $0.10 \pm 0.03$ & $39.33 \pm 19.60$ & $23.08 \pm 13.71$ & $37.58 \pm 20.52$ \\
\hline
\end{tabular}

*: Significantly different from negative control group $(\mathrm{P}<0.05 ; \mathrm{n}=6)$.

Generally, numbers of cells in lavage fluid are positively correlated with severity of silicosis. Thus, in conjunction group, but not significantly different from those for the IBCN inhalation groups. This implies that IBCN inhalation treatments may be effective for mitigating the cardiotoxicity of silica particle inhalation. There were no significant differences between the negative and positive control groups with respect to their average liver coefficients. However, there were significant differences $(\mathrm{P}<0.05)$ between the liver coefficients for the positive control group and IBCN inhalation groups, suggesting that IBCN inhalation may adversely affect the liver. The spleen coefficients for the negative and positive control groups did not differ significantly. However, there were significant differences ( $\mathrm{P}$ $<0.05$ ) between the spleen coefficients for the negative control group and $1 \%$ IBCN group, so the $1 \%$ IBCN treatment may adversely affect the spleen as well as the liver. The lung coefficients for all of the silica-exposed groups (i.e. the positive control group and all the IBCN inhalation groups) were significantly greater $(\mathrm{P}<0.05)$ than the coefficient for the negative control group. There were no significant differences between the lung coefficients for the three silica-exposed groups. These findings indicate that tracheal injection of silica particles causes lung tissue reproduction and silicosis. There were significant differences $(\mathrm{P}<0.05)$ between the kidney coefficients for the negative and positive control groups. However, there were no significant differences between the kidney coefficient of the negative control group and those for the IBCN inhalation groups. IBCN inhalation may thus partially alleviate the adverse effects of silica particle inhalation on the kidneys.
0.41 and $0.83 \pm 0.43 \times 10^{9}$ cells $/ \mathrm{ml}$, respectively). The cell counts in the lavage fluid from rats in the $1 \%$ IBCN inhalation and positive control groups were significantly higher $(\mathrm{P}<0.05)$ than the count for the negative control group, but not significantly different from those for the other IBCN inhalation groups. with the finding that the $1 \%$ IBCN inhalation treatment resulted in the highest average consolidated lung tissue area 
(Figure 2D-F), the high numbers of cells in the lung lavage fluid of the $1 \%$ I IBCN inhalation group indicate that this treatment had significantly stronger negative effects on the lung tissues than the $2 \%$ or $3 \%$ IBCN inhalation treatments. Typing of the counted cells in the lung lavage fluid obtained from each treatment group indicated that the vast majority were lymphocytes, neutral cells, or giant divinatory cells (Table 2). At the end of the treatment period, $\mathrm{N}$ cells were most abundant in the lavage fluid of the negative control group, followed by $\mathrm{M}$ and then $\mathrm{L}$ cells. Conversely, the lavage fluid of the silica-exposed groups was dominated by L cells, followed by $\mathrm{M}$ and then $\mathrm{N}$ cells. There were significant differences in proportions of cell types between the negative control group and the silica-exposed groups $(\mathrm{P}<0.05)$. These findings are both consistent with expectations and clinically relevant as $\mathrm{M}$ cells are phagocytes that are important for lung health, and their numbers are greatly reduced by dust exposure [1].

\subsection{Wet and Dry Lung Weights and Silica Clearance Rates in the Lungs of Silica-Exposed Rats}

Among the experimental groups, the total wet lung weight increased in the following order: negative control group < positive control group $<2 \%$ IBCN inhalation group $<3 \%$ IBCN exposure group $<1 \%$ IBCN exposure group (Table 3 ).
The differences in these weights between the negative control and silica-exposed groups, and between the $1 \%$ IBCN and positive control groups, were significant $(\mathrm{P}<0.05)$, but not those among the three IBCN inhalation groups. The total dry lung weights of the five groups increased in the same order as the wet lung weights (Table 3). Silica clearance rates from whole lungs, based on amounts of silica removed during lavage, ranged from $0.89 \pm 0.23$ to $1.06 \pm 0.48 \mathrm{mg} / \mathrm{d}$ for the three IBCN inhalation groups (Table 3 ). These rates are much higher than the negligible rates expected in the absence of treatment, and substantially higher than rates previously observed in rats treated by inhalation of kombucha preparations $(0.47 \pm 0.69 \mathrm{mg} / \mathrm{d})$ [1]. These findings have high potential clinical relevance as silica particles with diameters of 1-5 $\mu \mathrm{m}$ are relatively dense and very chemically stable, which greatly hinders their clearance from lungs. Thus, tracheal silica injection is widely used to simulate effects of silicosis in animals. Hence, IBCN inhalation promotes silica clearance in this rat silicosis model and could have considerable therapeutic potential. However, the silica clearance rate for the positive control group, treated with saline inhalation, was significantly greater $(2.24 \pm 0.47 \mathrm{mg} / \mathrm{d})$ than rates recorded for any of the IBCN inhalation groups ( $\mathrm{P}$ $<0.05)$.

Table 3. Effects of the IBCN inhalation and control treatments on silica removal from the lungs of rats exposed to silica dust particles (Mean \pm SD).

\begin{tabular}{lllll}
\hline Treatment & Total wet lung weight $(\mathbf{g})$ & Total dry lung weight $(\mathbf{g})$ & Silica removed from the lung (mg) & $\begin{array}{l}\text { Silica clearance rate for whole } \\
\text { lung (mg/d) }\end{array}$ \\
\hline 1\% IBCN exposure & $3.974 \pm 0.557^{*, \#}$ & $0.883 \pm 0.109^{*, \#}$ & $13.37 \pm 3.43^{\#}$ & $0.89 \pm 0.23^{\#}$ \\
$2 \%$ IBCN exposure & $3.484 \pm 0.757^{*}$ & $0.796 \pm 0.169^{*}$ & $16.49 \pm 4.54^{\#}$ & $1.10 \pm 0.30^{\#}$ \\
$3 \%$ IBCN exposure & $3.548 \pm 0.807^{*}$ & $0.829 \pm 0.181^{*}$ & $15.96 \pm 7.13^{\#}$ & $1.06 \pm 0.47^{\#}$ \\
Positive control & $3.045 \pm 0.920^{*}$ & $0.689 \pm 0.212^{*}$ & $33.53 \pm 7.06$ & $2.24 \pm 0.47$ \\
Negative control & $1.090 \pm 0.116^{\#}$ & $0.245 \pm 0.195^{\#}$ & -- & -- \\
\hline
\end{tabular}

* and \#: Significantly different from negative and positive control group, respectively $(\mathrm{P}<0.05 ; \mathrm{N}=6)$. --: Not applicable as rats in the negative control group were not exposed to silica.

\section{Discussion}

$\mathrm{BC}$ is an extremely pure form of cellulose that has several useful properties, including high purity and water-holding capacity, a three-dimensional nanofibrillar network, mechanical strength, biodegradability and biocompatibility [17]. It is therefore considered to have great potential as a nanomaterial in various high-tech contexts, including biomedical applications, most notably in controlled drug-delivery systems $[18,19]$. The aerodynamic diameters of the silica particles used in most silicosis models range from 0.5 to $5 \mu \mathrm{m}$. Because particles of this size are not readily transported from the lungs to other organs, they generally remain where they are deposited in the lungs or are cleared by the mucociliary system. Standard silica particles in this size range are therefore widely used to create rat models of silicosis caused by inhalation exposure. The results obtained in this work suggest that IBCN exposure promoted the clearance of silica particles from the lungs in a rat silicosis model. We have previously identified Chinese herbal kombucha inhalation as a potentially effective dust-removing probiotic treatment in the rat silicosis model used in this work. Spraying with herbal kombucha induced a whole lung silica clearance rate of $0.47 \pm 0.69 \mathrm{mg} / \mathrm{d}$ [1], similar to (but lower than) the rates observed in the $1 \%, 2 \%$ and 3\% IBCN exposure groups $(0.892 \pm 0.228,1.099 \pm 0.302$ and $1.063 \pm 0.476 \mathrm{mg} / \mathrm{d}$, respectively). This suggests that the BC secreted by G. xylinus in herbal kombucha may partially explain its positive effects on silica particle clearance. These results also indicate that IBCN and BC secreted by G. xylinus may be effective tools for adsorbing silica particles and increasing their rate of clearance from lungs. Accordingly, small particles of regenerated cellulose with diameters of 1-8 $\mu \mathrm{m}$ have previously been cationically modified and used as adsorbents to promote endotoxin removal [20].

Mucociliary clearance in the mammalian lung is an important innate defense mechanism that helps to clear inhaled pollutants, allergens, pathogens, and mucus secreted by the goblet cells and submucosal glands from the surfaces of the upper and lower airways [21]. The mucus clearance system consists of two layers. One is a mucus layer, also known as the gel layer, which traps inhaled particles and 
transports them out of the lung via a process driven by the cilia. The other is a periciliary layer, also known as the brush layer, which provides a favorable environment for ciliary beating and cell surface lubrication. The beating and relaxation of the cilia occur in the brush layer, which contains secreted and transudated molecules [21, 22]. Generally, the mucus clearance system is more capable of brushing inhalable particles larger than ca. $2.5 \mu \mathrm{m}$ from the lungs than smaller ones [23, 24]. This suggests that small particles may adhere more tightly to the mucus layer, thereby changing its physical properties (inter alia altering its rheological parameters, and increasing its viscoelasticity and adhesivity), thereby interfering with its normal structure and function. Moreover, small particles trapped in the mucus layer may subsequently move into the PCL, preventing their clearance by the mucus clearing system. However, adsorption of such small silica particles by G. xylinus cells or IBCN may effectively increase their size, thereby facilitating their clearance from the lungs. The results presented here demonstrate that IBCN exposure (particularly exposure to the $2 \%$ and $3 \%$ sprays) had some positive effects on the lungs and other organs in a rat silicosis model. These effects resembled those observed after treatment with herbal kombucha and included the mitigation of cardio- and kidney toxicity caused by silica injection, and reductions in cell counts in the lung lavage fluid of silica-exposed rats. However, IBCN exposure also had some adverse effects on rat organs, including the liver. Such effects were not observed after spraying with herbal kombucha, which may therefore be a better option for the practical treatment of silicosis. IBCN may function as a kind of artificial and moving periciliary layer and help to clear particles in the lung.

The difference in the results obtained with IBCN and kombucha suggests that the process of preparing IBCN yields $\mathrm{BC}$ with somewhat different physicochemical properties to that found in kombucha, and that IBCN may have some cytotoxic effects, or that other kombucha components have positively synergistic effects. No toxicity of BC nanofibers has been previously reported, but their uptake into cells could conceivably perturb various cellular processes, and thus be harmful to the lungs. In accordance with this hypothesis, the $1 \%$ and $3 \%$ IBCN inhalation treatments caused more extensive lung tissue consolidation and more indications of lung inflammation than the positive control treatment.

Unexpectedly, saline inhalation stimulated silica particle clearance in the rat silicosis model, at almost double the rates observed in the ICBN-treated rats $(2.24 \pm 0.47 \mathrm{mg} / \mathrm{d}$, versus $0.89 \pm 0.23$ to $1.06 \pm 0.48 \mathrm{mg} / \mathrm{d}$ ) and substantially higher rates than previously observed following treatment by herbal kombucha inhalation $(0.47 \pm 0.69 \mathrm{mg} / \mathrm{d})$ [1]. In fact, the silica particle clearance rate observed in the positive control group is, to our knowledge, the highest reported in any rat silicosis model. However, it should be noted that the results obtained in this work and our previous study on treatment with herbal kombucha may not be directly comparable because the kombucha was applied using a plastic sprayer whereas in the present study the saline solution and IBCN suspensions were applied using an ultrasonic nebulizer. In our previous investigation, the clearance rate achieved by saline inhalation via a plastic sprayer was $0.25 \pm 0.20 \mathrm{mg} / \mathrm{d}$ [1]. Thus, the switch from application via a plastic sprayer to an ultrasonic nebulizer increased the rate of silica particle clearance more than five-fold. It is possible that a similar increase in treatment effectiveness could be achieved by applying herbal kombucha using the ultrasonic nebulizer. This warrants further investigation. Because the silica clearance stimulated by IBCN inhalation appears to operate via a different mechanism to that activated by saline inhalation, it may be beneficial to combine both treatments to maximize the clearance of particulate matter from the lungs of long-term sufferers of silicosis.

\section{Discussion}

These results provide the first evidence for a functional effect of IBCN inhalation in a rat silicosis model, indicating that bacterial cellulose nanofiber inhalation can facilitate silica particle clearance. Further studies are required to determine whether these effects are mediated by IBCN and define the mechanisms involved. The findings also indicate that salt water may effectively clear dust from lungs, thereby alleviating risks of silicosis and reducing risks associated with haze and smog.

\section{Acknowledgements}

The authors thank Dr. Fei Wang and Qing Ding of the Research Institute of Agricultural Products Processing, Chinese Academy of Tropical Agricultural Sciences, for their excellent technical assistance in the analysis of IBCN and experimental data.

\section{References}

[1] Fu NF, Luo CH, Wu JC, Zheng YY, Gan YJ, Ling JA, Liang HQ, Liang DY, Xie J, Chen XQ, Li XJ, Pan RH, Chen ZX, Jiang SJ. Clearance of free silica in rat lungs by spraying with chinese herbal kombucha. Evid Based Complement Alternat Med. 2013: 790792.

[2] Fu NF, Wu JC, Fu QJ, Jiang SJ, Wu SC. Report of curing 43 gold mineral pneumoconiosis with Chinese herbal kombucha. J Aerospace Med 2013; 24: 987-989.

[3] Greenwalt CJ, Steinkraus KH, Ledford RA. Kombucha, the fermented tea: microbiology, composition, and claimed health effects. J Food Prot 2000; 63: 976-981.

[4] Argun ME, Dursun S, Ozdemir C, Karatas M. Heavy metal adsorption by modified oak sawdust: thermodynamics and kinetics. J Hazard Mater 2007; 141: 77-85.

[5] Chen S, Zou Y, Yan Z, Shen W, Shi S, Zhang X, Wang H. Carboxymethylated-bacterial cellulose for copper and lead ion removal. J Hazard Mater 2009; 161: 1355-1359.

[6] Lu M, Guan XH. Adsorption mechanism of bacterial cellulose to heavy metal ions. Chem Eng 2012; 40: 29-33. 
[7] Oshima T, Kondo K, Ohto K, Inoue k, Baba Y. Preparation of phosphorylated bacterial cellulose as an adsorbent for metal ions. React Funct Polym 2008; 68: 376-383.

[8] Tang SJ, Yang XX and Hong F. Production of bacterial cellulose by Kombucha. J Cellulose Sci Tech 2012; 20: 40-45.

[9] Zhou H, Sun DP, Zhu CL, Xu CY. Study on the adsorption of noxious substances in human body by bacterial cellulose. $\mathrm{J}$ Cellulose Sci Tech 2009; 17: 41-46.

[10] Zhu CL, Li F, You QS. Preparation of nanometer biomaterial bacterial cellulose and observation of its ultra-structure. J Biomed Engineering Res 2008; 27: 287-290.

[11] Tazi N, Zhang Z, Messaddeq Y, Almeida-Lopes L, Zanardi LM, Levinson D, Rouabhia M. Hydroxyapatite bioactivated bacterial cellulose promotes osteoblast growth and the formation of bone nodules. AMB Express 2012; 2: 61.

[12] Chen ZP, Su WG, Yan S. Therapeutic effect of Tetrandrine on patients with pneumoconiosis. J Clini Pulmonary Med 2012; 17: $1226-1227$.

[13] Costantini LM, Gilberti RM, Knecht DA. The phagocytosis and toxicity of amorphous silica. PLoS One 2011; 6: e14647.

[14] Costola-de-Souza C, Ribeiro A, Ferraz-de-Paula V, Calefi AS, Aloia TPA, João Antonio Gimenes-Júnior JA, Izidio de Almeida V, Lobão Pinheiro M, Palermo-Neto J. Monoacylglycerol lipase (MAGL) inhibition attenuates acute lung injury in mice. PLoS One 2013; 8: e77706.

[15] Davis GS, Pfeiffer LM, Hemenway DR, Rincon M. Interleukin-12 is not essential for silicosis in mice. Part Fibre Toxicol 2006; 3: 2 .

[16] Davis JM, Addison J, Bolton RE, Donaldson K, Jones AD,
Smith T. The pathogenicity of long versus short fibre samples of amosite administered to rats by inhalation and intraperitoneal injection. Br J Exp Pathol 1986; 67: 473-491.

[17] Lin WC, Lien CC, Yeh HJ, Yu CM, Hsu SH. Bacterial cellulose and bacterial cellulose-chitosan membranes for wound dressing applications. Carbohydr Polym 2013; 94: 603-611.

[18] Abeer MM, Mohd Amin MC, Martin C. A review of bacterial cellulose-based drug delivery systems: their biochemistry, current approaches and future prospects. J Pharm Pharmacol 2014; 66: 1047-1061.

[19] Silvestre AJ, Freire CS, Neto CP. Do bacterial cellulose membranes have potential in drug-delivery systems? Expert Opin Drug Deliv 2014; 21: 1-12.

[20] Weber C, Henne B, Loth F, Schoenhofen M, Falkenhagen D. Development of cationically modified cellulose adsorbents for the removal of endotoxins. ASAIO J 1995; 41: 430-434.

[21] Button B, Cai LH, Ehre C, Kesimer M, Hill DB, Sheehan JK, Boucher RC, Rubinstein M. Periciliary brush promotes the lung health by separating the mucus layer from airway epithelia. Science 2012; 337: 937-941.

[22] Puchelle E, De Bentzmann S, Zahm JM. Physical and functional properties of airway secretions in cystic fibrosis--therapeutic approaches. Respiration 1995; 62: 2-12.

[23] Geiser M, Kreyling WG. Deposition and biokinetics of inhaled nanoparticles. Part Fibre Toxicol 2010; 7: 2.

[24] Oberdörster G, Ferin J, Lehnert BE. Correlation between particle size, in vivo particle persistence, and lung injury. Environ Health Perspect 1994; 102: 173-179. 